\title{
Continuous Monitoring of Comet Holmes from Before the 2007 Outburst
}

\author{
Ehab E. El-Houssieny ${ }^{1}$ • Robert J. Nemiroff ${ }^{1} \bullet$ \\ Timothy E. Pickering ${ }^{2}$
}

(C) Springer-Verlag $\bullet \bullet \bullet \bullet$

Not to appear in Nonlearned J., 45.

Abstract The outburst and subsequent brightness evolution of Comet 17P/Holmes has been observed using the MMT Observatory's All-Sky Camera (Pickering 2006) on Mt. Hopkins near Tucson, Arizona, USA. The comet was picked up at the limiting visual magnitude of 5.5 on October 24.38 and tracked by the camera continuously until sunrise four hours later. During this time the comet brightened to visual magnitude 3.5. Comet Holmes was next observed just after sunset on October 25.23 at visual magnitude 2.5 where it remained approximately constant over the next three days. The comet then began to dim slowly and was followed into the early months of 2008 with periods of dense time coverage.

Keywords comet Holmes; brightness variation; the total magnitude formula

\section{Introduction}

Comets have been noted in the sky for almost as long as history has been recorded. A verifiable brightness variation for any bright comet is rare, however, since human observers are hard to calibrate, photographic magnitudes can be unreliable for bright objects, and because telescopes with modern CCDs typically have fields of view too small to contain a comet that has reached naked-eye visibility. Even for comets monitored

Ehab E. El-Houssieny

Robert J. Nemiroff

Michigan Technological University, Department of Physics, 1400 Townsend Drive, Houghton, MI 49931, USA

Timothy E. Pickering

MMT Observatory, University of Arizona, Tucson, AZ 85721, USA

by CCD, unusual cometary events are rarely recorded, since observers are typically alerted to look only after such an event has occurred.

In recent years, however, a class of all-sky cameras has begun to be used routinely in astronomy, primarily to assess sky conditions (Nemiroff and Rafert 1999; Pickering 2006; Shamir and Nemiroff 2005a,b). These cameras typically utilize fisheye lenses with fields of view in excess of 150 degrees and can thus capture images of a comet of almost any size. Furthermore, these cameras typically operate every clear night, and so are likely to be operating during an unusual cometary outburst.

In 2007 October, periodic comet Holmes underwent an unusual outburst which increased its brightness from an apparent visual magnitude of about 17 to near 3 . This paper reports details from an all-sky camera that captured this cometary outburst and monitored the brightness of the comet continuously for the next few hours and for many nights over the next three months.

\section{A Brief History of Comet Holmes}

Comet Holmes was discovered by Edwin Holmes (London, England) on November 6, 1892 within the Andromeda Galaxy (M31). The comet brightened from dimmer than visual magnitude 17 to about visual magnitude 2.8 over about 42 hours, creating a coma about 5 arc minutes diameter (Whipple 1984). The comet's discovery was confirmed by Edward Walter of the Royal Observatory in Greenwich, England Whipple 1984; Bobrovnikoff 1943). Unexpectedly, comet Holmes underwent a second outburst only few months later, on January 16, 1893. In the second outburst, the comet brightened to visual magnitude 8 and exhibited a coma of 41 arc seconds in diameter. The comet steadily exhibited a larger coma until late the next night and 
then steadily faded after the next outburst. The last observation before fading from visibility was made by H. C. Wilson of Goodsell Observatory in Northfield, Minnesota on April 4, 1893 (Bobrovnikoff 1943; Zwiers 1912). The comet was lost after 1906 until being reacquired on July 16, 1964 by Elizabeth Roemer of the Naval Observatory in Arizona, USA (Whipple 1984).

Several attempts have been made to determine the comet's orbital elements. The first orbit determination was calculated by H. C. Kreutz using three positions measured on November 9, 10, and 11, 1892 (Zwiers 1912). Kreutz discussed the difficulty of calculating comet Holmes' orbit and introduced four potential parabolic orbits satisfying three observations with perihelion passage time ranging from February 28 June 7, 1892 and an orbital period of 6.9 years. During the next few weeks, several more attempts were made to more precisely determine comet Holmes' orbit. These attempts also derived rather different orbital elements with perihelion passage estimates ranging from February 28 to August 16, 1892.

\section{The Outburst and the Brightness Variation of Comet Holmes in 2007-2008}

On October 24, 2007, comet Holmes underwent an outburst similar to its first outburst. During the early hours of October 24, 2007, the comet became much brighter, increasing its brightness from a visual magnitude of about 18 to 2.5 over less than two days. Comet Holmes became the third brightest object in the constellation of Perseus (see Figure 1-b) and was visible to the unaided eyes of even casual observers.

Although hampered by moonlight, the MMT AllSky Camera was able to capture comet Holmes on the night of its sudden brightening. Through the course of its normal operation, the All-Sky Camera was then able to follow the evolution of comet Holmes for several months after its outburst. On a dark night, the system defaults to an 8.533 second exposure time which results in a limiting magnitude of about 5.5 in $\mathrm{V}$ and 6 in $\mathrm{R}$. Sensitivity is decreased by moonlight, however, due to glare, reduced gain, and reduced exposure time.

It was necessary to divide comet Holmes' brightness variation into two graphs according to our continuous observations of the comet from the time it became visible on MMTO All Sky Camera images over the next three months. Figure 2 shows a plot of the visual magnitude of comet Holmes over the first three days where the comet exhibited extreme magnitude change from below visibility to magnitude 2.5 in less than 24 hours. Data points are plotted for

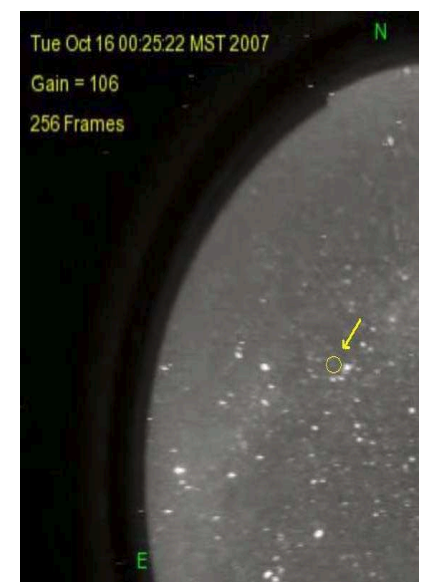

(a)



(b)
Fig. 1 Two images extracted from MMTO All Sky Camera image archive. Comet Holmes is barely visible on the image on the left, taken on October 16 when it was fainter than 17th magnitude, while only 18 nights later, at the same sidereal time, the comet is easily visible on the image on the right at a visual magnitude of 2.8 .

every hour by averaging over each 10-second exposure. While Figure 3 shows a plot of the comet magnitude over the next three months where the comet exhibits steady fading phase. Data points are plotted for every notable magnitude change, nearly for every 24 hours. Magnitude estimates in both graphs were made by comparison to stars of cataloged magnitudes (Henry Draper Catalogue at Harvard College Observatory)

Figure 2 shows that comet Holmes started its outburst at the early hours of October 24, 2007, in particular about 9.5 Universal Time (UT) and it was of magnitude 5.5 to be visible to unaided eyes. In less than 24 hours, comet Holmes surprisingly brightened to magnitude 3.5 that is enough to be seen in full-moon night and town light pollution. In the next night, October 25, 2007, at 06:53 am UT, the comet reached magnitude 2.5. This is followed by complete constancy at maximum light sustained for 2 days after the comet outburst.

Figure 3 shows that comet Holmes remained visible over the next three months. Night to night, from October 27, 2007 - January 1, 2008, comet Holmes is slowly and steadily fading through periods of about three days long. 


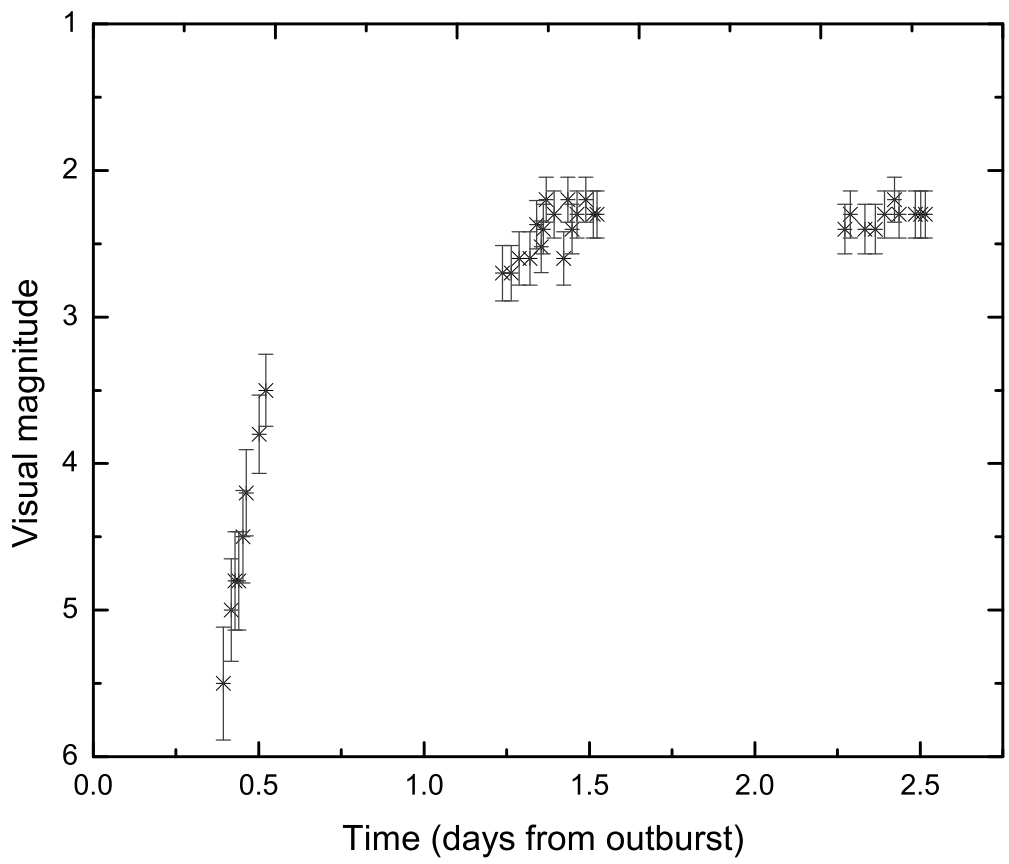

Fig. 2 The Comet Holmes' brightness variation from October 24-26, 2007.

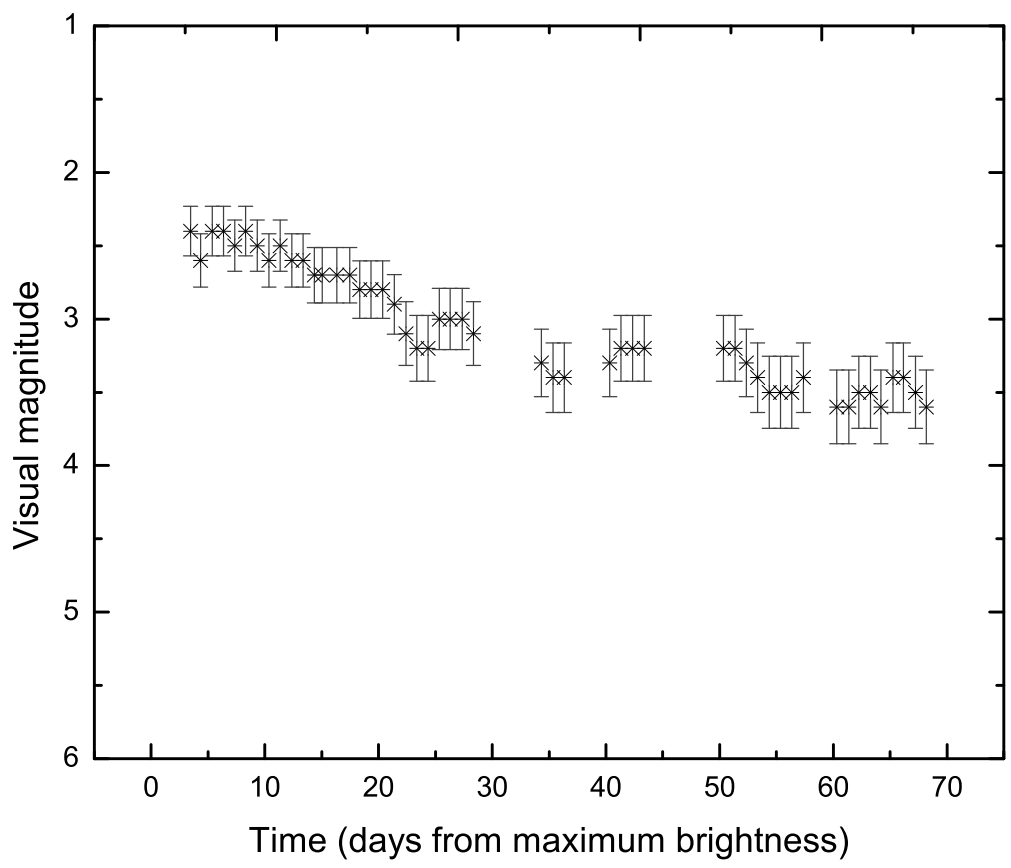

Fig. 3 The Comet Holmes' brightness variation from October 27, 2007 - January 1, 2008. 


\section{Quantifying Comet Holmes' Brightness} Variation Curve

For explanatory and future predictive value of the photometric parameters, an attempt was made to quantify the evolution of the brightness of comet Holmes as a function of the heliocentric and geocentric distances. It is well known that the dust jets of active comets may take tens of days to dissipate by solar radiation pressure into a comet's tail, especially at large heliocentric distances, and there might be an unknown contribution of a comet's tail at small phase angles because it lies on the line-of-sight of the comet (Gonzales 1969; Ip, W.-H. 1979). The 2007 outburst of comet Holmes occurred at relatively large heliocentric distance and almost zero phase angle. During the first month from the outburst the comet dimmed rapidly with dimming rate of 0.02 magnitude/day, while after November 23, 2007 , the comet experienced a slow and steady dimming phase with dimming rate of 0.0075 magnitude/day, see Figure 4 This slow and steady dimming phase might be interpreted as due to the increase in the heliocentric distance because the short term dust dissipation constitutes a small fraction of the total brightness variation. Such reasoning along with precise total brightness estimates taken by single instrument with small aperture, such as MMT all-sky camera (aperture $<1 \mathrm{~cm}$ ), may allow the use of the approximated equation of the total magnitude of a comet with coma, represented by

$$
m=H_{0}+5 \log (\Delta)+2.5 n \log (r),
$$

or by rearranging the terms to be in the form,

$$
m-5 \log (\Delta)=H_{0}+2.5 n \log (r),
$$

where $H_{0}$ is the absolute magnitude of the comet, $\Delta$ and $r$ are the geocentric and the heliocentric distances (AU) respectively, and $n$ is the exponent of the heliocentric distance power-law (Fernandez, 2005).

To investigate the variation of the photometric parameters $H_{0}$ and $n$ of comet Holmes, we have plotted the linear equation (11) and used linear fitting in an attempt to minimize the coma and the outburst consequences. Figure [5]shows that there is a phase transition had occurred on about November 23, 2007, that might be interpreted as the beginning of the normal phase of the brightness variation of a comet, in which the brightness variation depends on the geocentric and the heliocentric distances as well as the phase angle according to equation (11). The resulted photometric parameters are summarized in Table (1).

\section{Summary and Conclusions}

Comet outbursts were discovered many decades ago (Bobrovnikoff 1943; Zwiers 1912), though a complete understanding as to why some comets under an outburst has remained elusive. However, comet brightness variation curves provide important information about comet flares (see, for example, Churyumov and Filoneko 1993). In an attempt to provide a clearer picture about this unusual cometary flare, our paper summarizes and analyzes the results of observations of comet Holmes' flare that began in October 2007. These observations were taken with the MMT All Sky Camera over three months from the beginning of its outburst. From the time comet Holmes became visible to the MMTO All Sky Camera, it brightened from visual magnitude 5.5 to magnitude 2.5 in less than 29 hours, and then followed by constancy at maximum brightness over the next two days. After reaching its peak, comet Holmes steadily faded through periods of about 3 days long to fainter magnitudes over the next three months to reach magnitude 3.7 on January 1, 2008. There is a phase transition had occurred on about November 23,2007 , in which the dimming rate was much smaller (0.0075 magnitude/day) and the photometric parameters was dramatically changed.

To the best of our knowledge, no comet has ever been monitored by a single dedicated instrument for so long a period of time previously. The MMT All Sky Camera has proved useful in that it can continuously monitor the variation of a comet's total visual brightness. It is our hope that the comet Holmes' brightness variation curves presented here will provide useful constraints for future comet outburst models.

\section{Acknowledgements}

We are grateful to the anonymous referees for valuable comments and suggestions that greatly improved our manuscript in all aspects. 


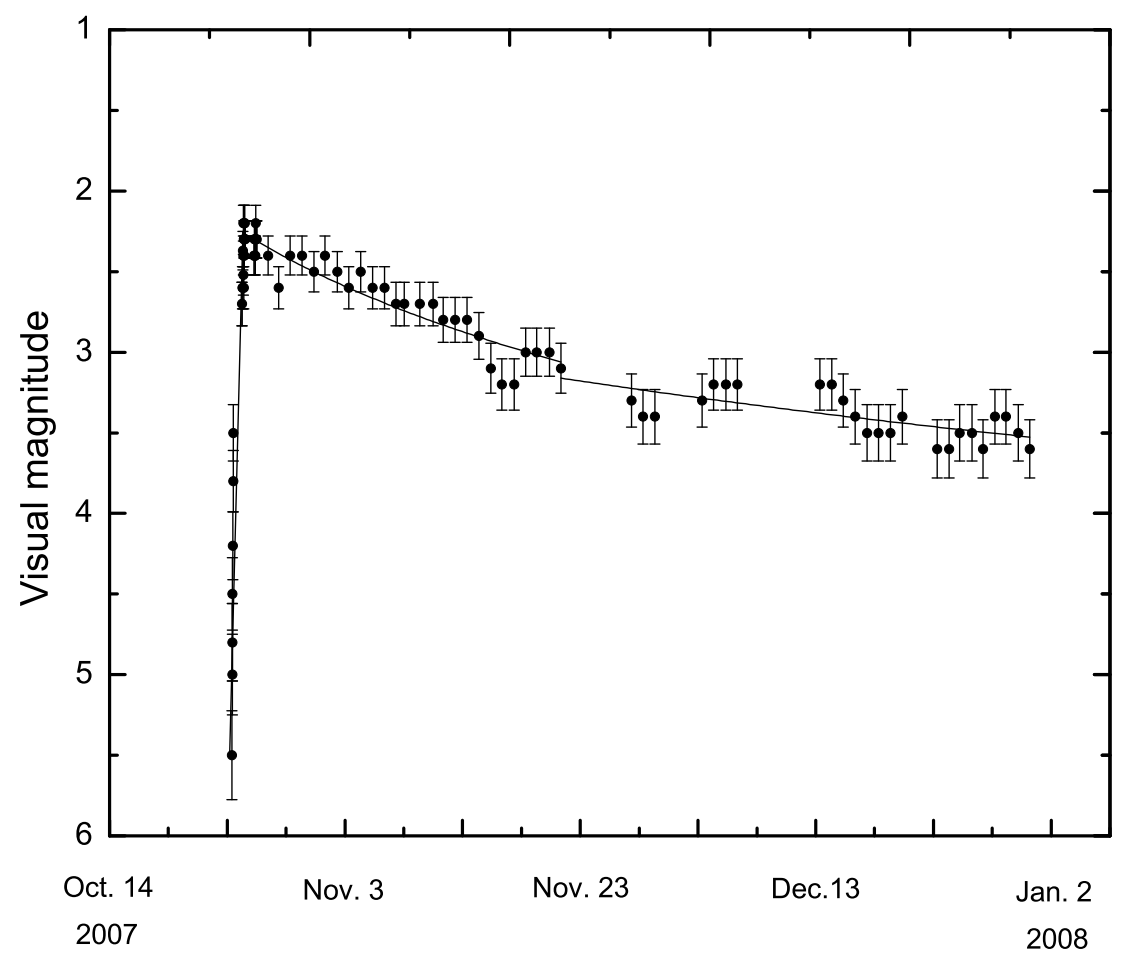

Time

Fig. 4 Comet Holmes brightness variation curve according to magnitude estimates is drawn by dots and the solid line represents the secular variation of the brightness fitted by the linear least square method.

Table 1 The photometric parameters of comet 17P/Holmes after its 2007 outburst.

\begin{tabular}{ccc}
\hline \hline Time range & Range of r (AU) & $H_{0}+2.5 n \log (r)$ \\
\hline Oct. 24 - Nov. 23, 2007 & $2.436-2571$ & \\
Nov. 23, 2007 - Jan. 1, 2008 & $2.571-2.717$ & $1.13( \pm 0.76387)+2.25( \pm 1.81734) \log (r)$ \\
\hline
\end{tabular}




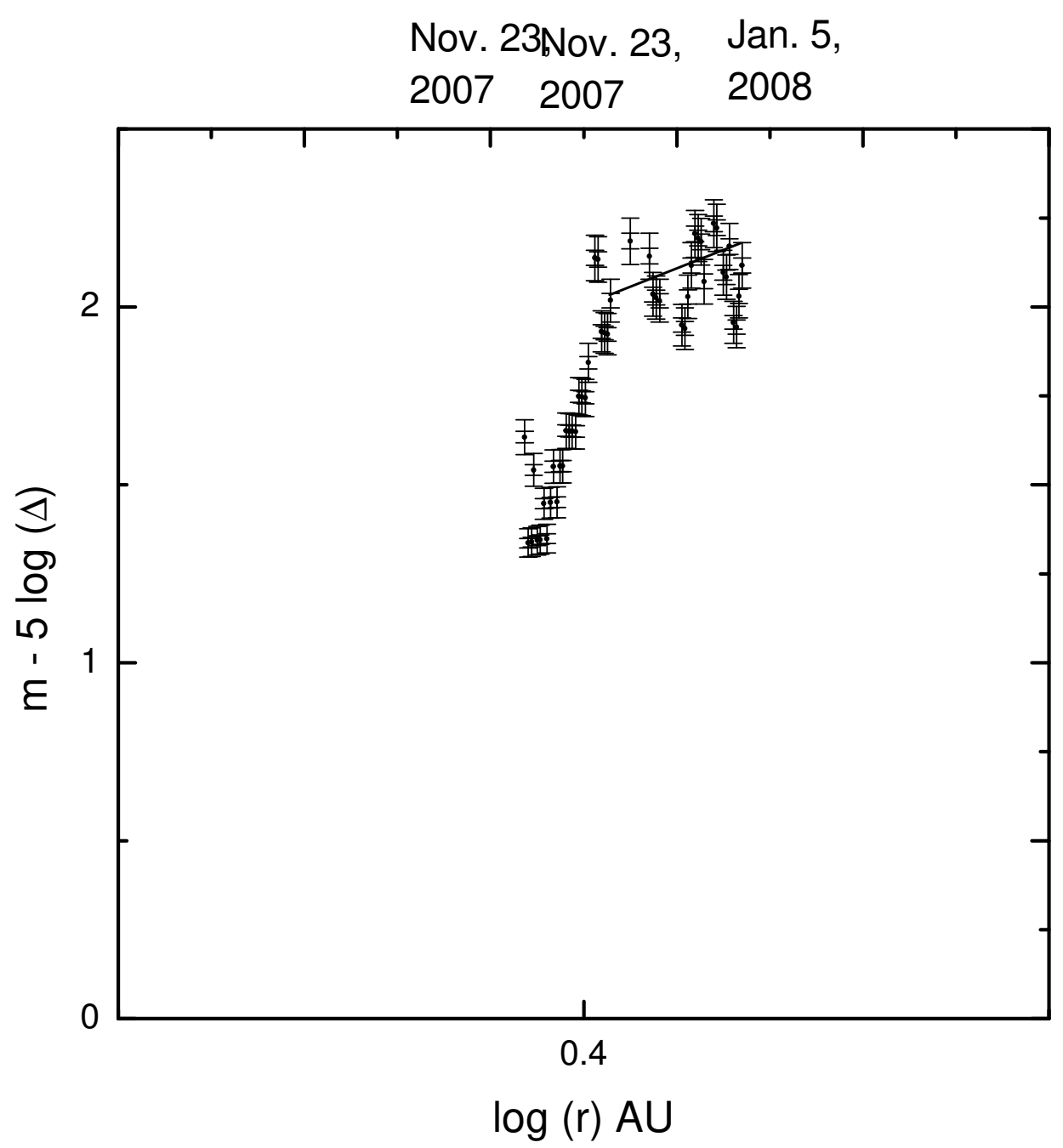

Fig. 5 The total brightness variation of comet 17P/Holmes with the linear fit representing the solution to equation (1). 


\section{References}

Bobrovnikoff, N. T.: The Periodic Comet Holmes (1892 III). PA, 51, 542B (1943)

Churyumov, K. I. and Filoneko, V. S.: Phase Dependencies of Cometary Light Curves. Abstracts of IAU Symp. 160: Asteroids, Comets, Meteors, Belgirate (Novara), Italy, P. 66, June 14-18 (1993)

Comet for Windows software by Seiichi Yoshida (1995-2004)

Gonzales, D. E.: Note on the solar wind-induced drag on comets. Solar Physics, Volume 9 (1969)

Henry Draper Catalogue, was compiled by Annie Jump Cannon and co-workers at Harvard College Observatory under the supervision of Edward C. Pickering.

Ip, W.-H. and Mendis D. A.: The structure of cometary ionospheres 2. CO-rich comets. Icarus 30, pp. 377-384 (1977)

Nemiroff, R. J. and Rafert, J. B.: Toward a Continuous Record of the Sky. PASP, 111, 886-897 (1999)

Pickering, T. E.: The MMT All-Sky Camera. SPIE 6267 (2006)

Shamir, L. and Nemiroff, R. J.: All-Sky Relative Opacity Mapping Using Nighttime Panoramic Images. PASP, 117, 835, 972-977 (2005)
Shamir, L. and Nemiroff, R. J.: PHOTZIP: A Lossy FITS Image Compression Algorithm that Protects User Defined Levels of Photometric Integrity. 129, 1, 539-546 (2005)

Whipple, F. L.: Comet 17P/Holmes, 1892III: A case of duplicity? Icarus, 60, issue 3 (1984)

Zwiers, H. J.: Researches on the orbit of the periodic comet Holmes and on the perturbations of its elliptic motion. De Roever Krober \& Bakels (1912) 